\title{
Molecular cloning and sequence analysis of the clorobiocin biosynthetic gene cluster: new insights into the biosynthesis of aminocoumarin antibiotics
}

\author{
Florence Pojer, Shu-Ming Li and Lutz Heide
}

Eberhard-Karls-Universität Tübingen, Pharmazeutische Biologie, Auf der Morgenstelle 8, D-72076 Tübingen, Germany Author for correspondence: Shu-Ming Li. Tel: +49 7071 2976995. Fax: + 497071295250.
e-mail: shuming.li@uni-tuebingen.de

\begin{abstract}
The biosynthetic gene cluster of the aminocoumarin antibiotic clorobiocin was cloned by screening of a cosmid library of Streptomyces roseochromogenes DS 12.976 with two heterologous probes from the novobiocin biosynthetic gene cluster. Sequence analysis revealed 27 ORFs with striking similarity to the biosynthetic gene clusters of novobiocin and coumermycin $A_{1}$. Inactivation of a putative aldolase gene, cloR, by in-frame deletion led to the abolishment of the production of clorobiocin. Feeding of the mutant with 3-dimethylallyl-4hydroxybenzoic acid (Ring A of clorobiocin) restored clorobiocin production. Here, it is suggested that the formation of Ring A of clorobiocin may proceed via a retro-aldol reaction catalysed by CloR, i.e. by a mechanism different from the previously elucidated benzoic acid biosynthetic pathway in Streptomyces maritimus. A comparison of the gene clusters for clorobiocin, novobiocin and coumermycin $A_{1}$ showed that the structural differences between the three antibiotics were reflected remarkably well by differences in the organization of their respective biosynthetic gene clusters.
\end{abstract}

Keywords: coumermycin $\mathrm{A}_{1}$, novobiocin, aldolase, retro-aldol reaction

\section{INTRODUCTION}

Aminocoumarin antibiotics, such as novobiocin, clorobiocin and coumermycin $\mathrm{A}_{1}$ (Fig. 1) (Berger \& Batcho, 1978), are produced by various Streptomyces strains and are very potent against Gram-positive pathogenic bacteria, including methicillin-resistant Staphylococcus strains. Novobiocin is licensed in the USA as an antibiotic for use in humans (Albamycin; Pharmacia $\&$ Upjohn).

Bacterial DNA gyrase is the target of the aminocoumarin antibiotics (Hooper et al., 1982; Maxwell, 1999). X-ray crystallographic examinations (Ali et al., 1993; Lewis et al., 1996; Maxwell, 1993; Tsai et al., 1997; Lafitte et al., 2002) have demonstrated that as well as the aminocoumarin moieties and the substituted deoxysugar moieties, the prenylated 4-hydroxybenzoate moieties of clorobiocin and novobiocin are essential for the binding of these compounds to the B subunit of gyrase.

The affinity of the aminocoumarin antibiotics for

The GenBank accession number for the sequence of cosmid K1F2 is AF329398. bacterial gyrase is extremely high. The inhibition constant $\left(K_{\mathrm{i}}\right)$ values of these antibiotics are in the $10 \mathrm{nM}$ range, i.e. two orders of magnitude lower than those of modern fluoroquinolones. This makes the aminocoumarins very interesting starting materials for the development of new antibacterial compounds.

Previously, our group has identified the biosynthetic gene clusters for novobiocin from Streptomyces spheroides NCIMB 11891 (Steffensky et al., 2000a) and for coumermycin $\mathrm{A}_{1}$ from Streptomyces rishiriensis DSM 40489 (Wang et al., 2000). In the present study, we report the identification of the clorobiocin biosynthetic gene cluster from Streptomyces roseochromogenes DS 12.976. A comparison of the gene clusters for clorobiocin, novobiocin and coumermycin $\mathrm{A}_{1}$ showed that the structural differences between the three antibiotics corresponded well to the differences in the organization of their respective biosynthetic gene clusters. Furthermore, the very stringent organization of the biosynthetic genes encoding the three different aminocoumarin antibiotics into 'modules', each of which carries the complete genetic information required for the biosynthesis of the respective antibiotic, offers excellent prospects for 
A
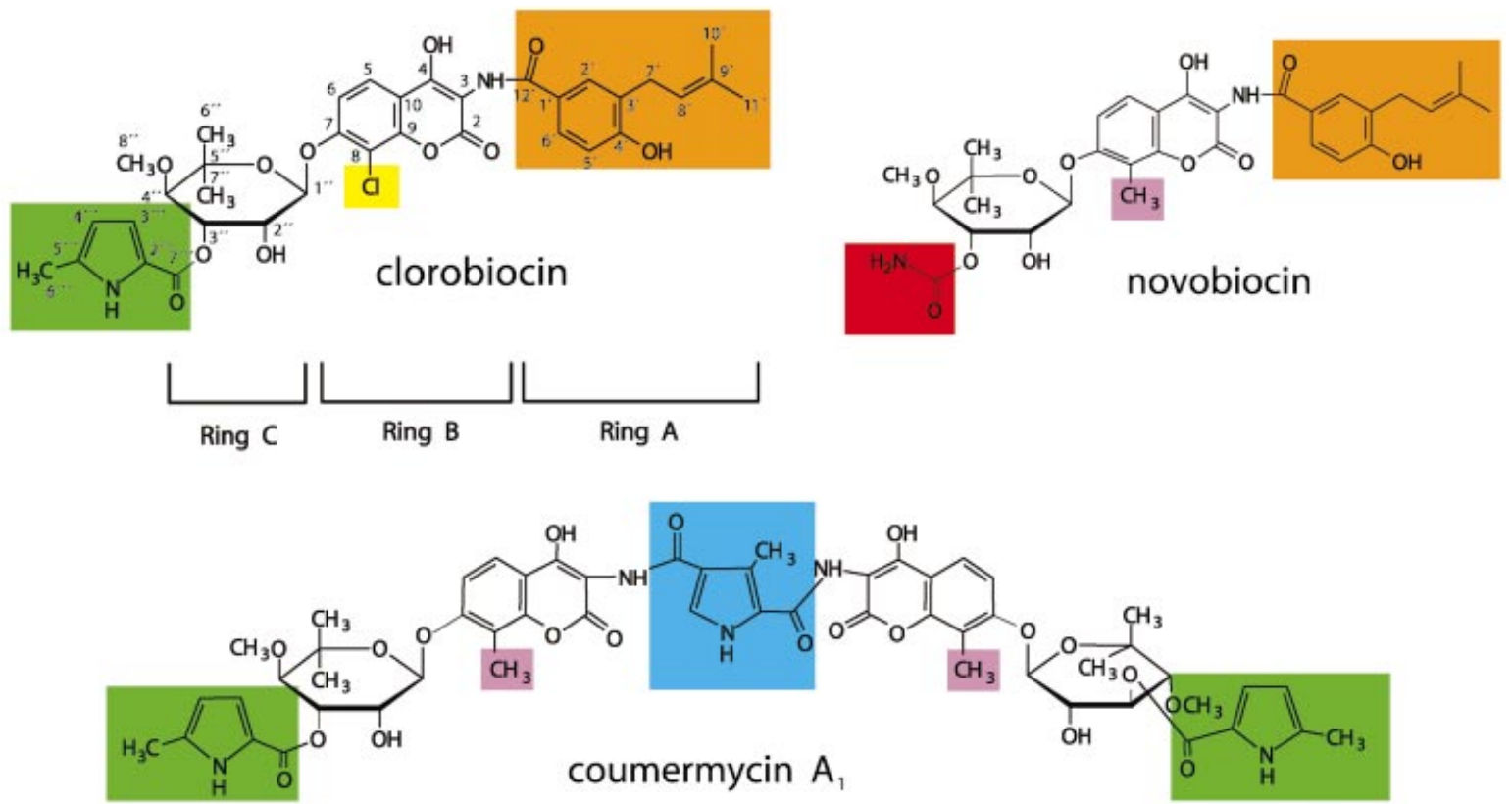

B

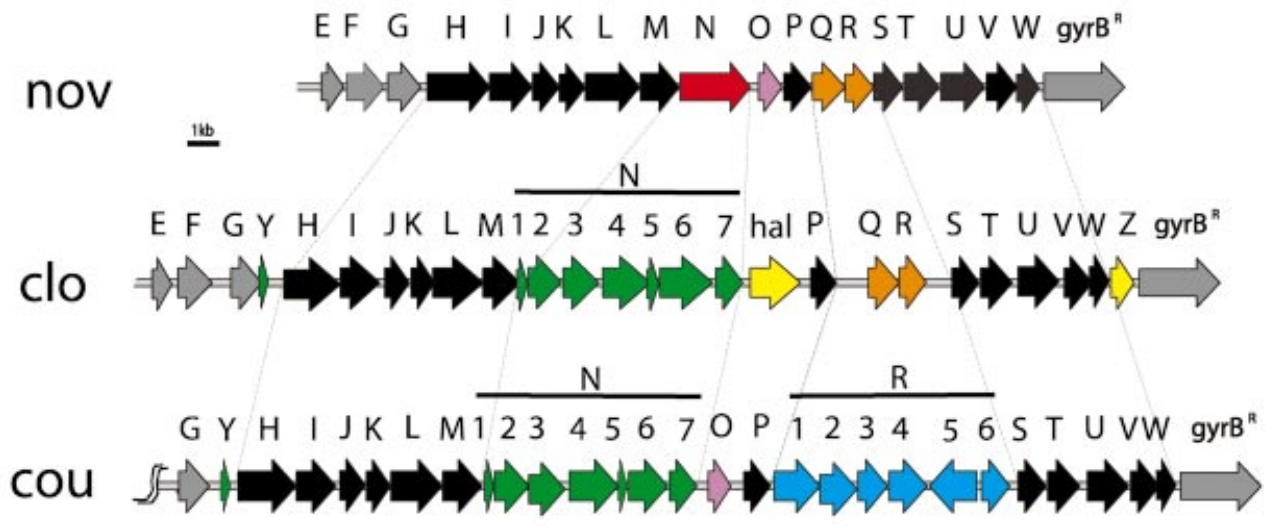

Fig. 1. (A) Structures of the aminocoumarin antibiotics. (B) Map of the clorobiocin biosynthetic gene cluster of $S$. roseochromogenes DS 12.976 (clo), compared to the biosynthetic gene clusters of novobiocin (nov) and coumermycin $A_{1}$ (cou). We have previously revised the nomenclature of the genes involved in coumermycin $A_{1}$ biosynthesis (GenBank accession no. AF235050).

the production of novel aminocoumarins using a combinatorial biosynthetic approach.

\section{METHODS}

Bacterial strains, plasmids and culture conditions. S. roseochromogenes var. oscitans DS 12.976 (kindly provided by Aventis) was routinely cultivated at $28{ }^{\circ} \mathrm{C}$ for 2 days in HA medium containing $1.0 \%$ malt extract, $0.4 \%$ yeast extract, $0 \cdot 4 \%$ glucose and $1.0 \mathrm{mM} \mathrm{CaCl}_{2}(\mathrm{pH} 7 \cdot 3)$. For the production of clorobiocin and other secondary metabolites, wild-type and mutant strains of $S$. roseochromogenes were cultured in $500 \mathrm{ml}$ baffled flasks containing $50 \mathrm{ml}$ pre-culture medium (Mancy et al., 1974). After growth of these cultures for $48 \mathrm{~h}$ at $33^{\circ} \mathrm{C}$ and 210 r.p.m., $5 \mathrm{ml}$ of the cultures were inoculated into $500 \mathrm{ml}$ baffled flasks containing $50 \mathrm{ml}$ production medium (Mancy et al., 1974). After inoculation into the production medium, cells were cultured at $33^{\circ} \mathrm{C}$ and 210 r.p.m. for 7 days.

Escherichia coli XL-1 Blue $\mathrm{MRF}^{\prime}$ (Stratagene) was grown in liquid or on solid Luria-Bertani medium at $37^{\circ} \mathrm{C}$ (Sambrook \& Russell, 2001). SuperCos-1 was purchased from Stratagene. pBSKT, an integrative vector carrying carbenicillin and thiostrepton resistances, was described by Lombo et al. (1997). Carbenicillin $\left(50 \mu \mathrm{g} \mathrm{ml}^{-1}\right)$ and thiostrepton $\left(50 \mu \mathrm{g} \mathrm{ml}^{-1}\right)$ were 
(a)

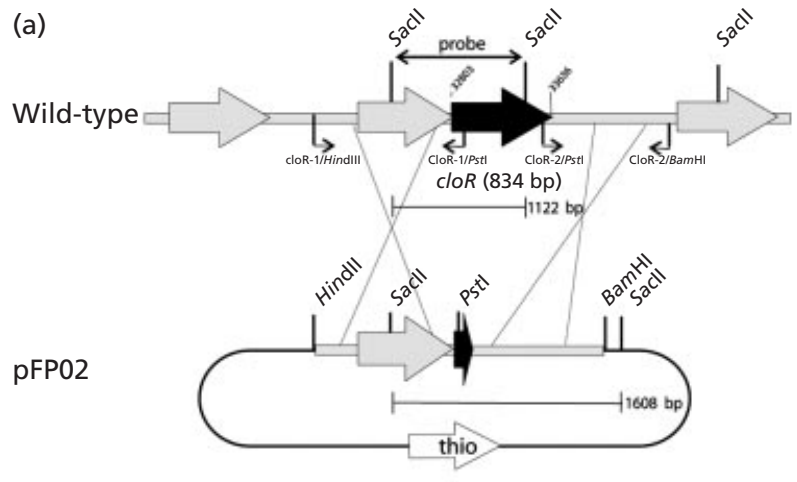

double-cross-over recombination

cloR-defective mutant

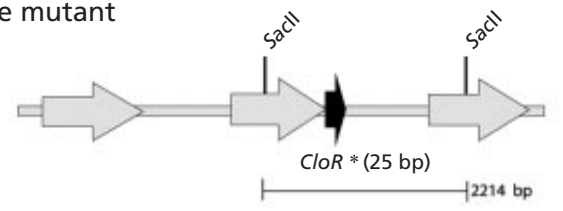

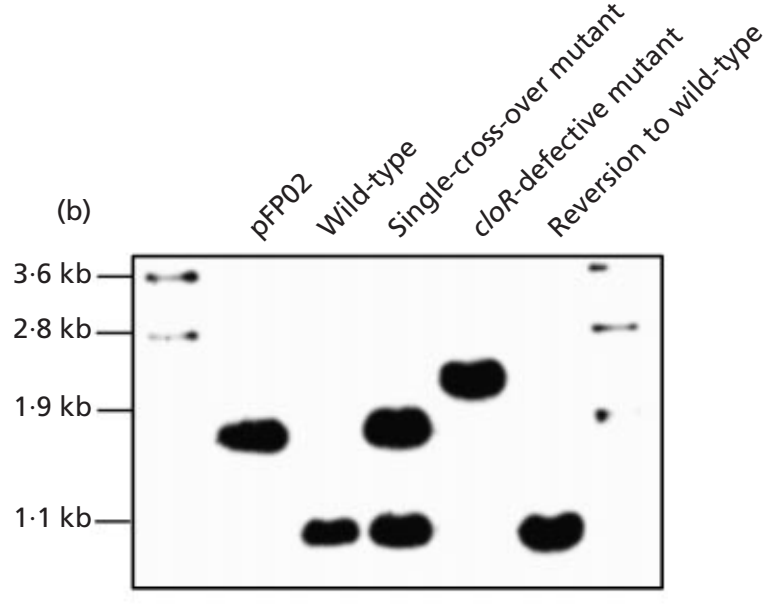

Fig. 2. Inactivation of $c l o R$ of the clorobiocin biosynthetic gene cluster. (a) Schematic presentation of the gene inactivation experiment. thio, Thiostrepton-resistance gene. (b) Southern blot analysis of the wild-type and mutant strains. Genomic DNA was digested with Sacll. The indicated 1122 bp Sacll fragment containing part of cloR in (a) was used as the probe.

used in the medium for selection of recombinant plasmids and strains.

Genetic procedures. Standard methods for DNA isolation and manipulation were performed as described by Kieser et al. (2000). DNA fragments were isolated from agarose gels using a NucleoSpin 2 in 1 extraction kit (Macherey-Nagel). Isolation of cosmids and plasmids was carried out with ion-exchange columns (Nucleobond AX kit; Macherey-Nagel). Genomic DNA was isolated from Streptomyces strains by lysozyme treatment and phenol/chloroform extraction.

Construction and screening of the cosmid library. Chromosomal DNA of $S$. roseochromogenes was partially digested with Sau3AI, dephosphorylated and then ligated into the BamHI sites of SuperCos-1. The ligation products were packaged with Gigapack III XL (Stratagene, Heidelberg; Germany) and transduced into E. coli XL-1 Blue MRF'.

Southern blot analysis was performed on Hybond-N membranes (Amersham) with digoxigenin-labelled probes by using the DIG high prime DNA labelling and detection kit II (Roche Molecular Biochemicals). Two probes, one containing part of the dTDP-glucose 4,6-dehydratase gene novT (Steffensky et al., 2000a) and one containing a $1.58 \mathrm{~kb} S p h \mathrm{I}-B a m \mathrm{HI}$ fragment of the novobiocic acid synthetase gene novL (Steffensky et al., 2000b), were used for hybridizations.

DNA sequencing and computer-assisted sequence analysis. Double-stranded sequencing of the entire cosmid K1F2 (carrying an insert of $42291 \mathrm{bp}$ ) was performed by the dideoxynucleotide chain termination method on a LI-COR automated sequencer (MWG-Biotech) using a shotgun library with DNA fragments of approximately $1.5-2.0 \mathrm{~kb}$ in length.

The DNASIS software package (version 2.1; Hitachi Software Engineering) was used for sequence analysis. Amino acid sequence homology searches were carried out in the GenBank database by using the BLAST program (release 2.0).

Construction of pFP02 for in-frame gene inactivation. For inactivation of cloR in $S$. roseochromogenes, the fragments cloR-1 (1282 bp) and cloR-2 (1301 bp) were amplified by PCR. Primer pair cloR-1/HindIII (5'-GTCACCGGAAGCTTTGCCTG-3') and cloR-1/Pst I (5'-GCATGTTCTGCAGAGCCTTG-3') was used to amplify cloR-1; primer pair cloR-2/Pst I (5'-GCCTGCACTGCAGGCCCCAA-3') and cloR-2/BamHI (5'-TCGTAGGATCCTCCCGTCGTC-3') was used to amplify cloR-2. Restriction sites introduced into the primers are shown in bold in the aforementioned sequences. cloR-1 was digested with HindIII and PstI and cloned into the corresponding sites of vector pBSKT, a pBluescript $\mathrm{SK}(+)$ derivative containing carbenicillin and thiostrepton resistances, resulting in pFP01. cloR-2 was digested with Pst I and $\mathrm{BamHI}$ and ligated into the same sites of pFP01 to give pFP02.

Transformation of $S$. roseochromogenes and selection of recombinant mutants. Transformation of $S$. roseochromogenes with pFP02 was carried out by polyethylene glycolmediated protoplast transformation (Kieser et al., 2000). For the preparation of protoplasts, mycelia of S. roseochromogenes were grown in CRM medium containing $10.3 \%$ sucrose, $2 \cdot 0 \%$ tryptic soy broth, $1.0 \% \mathrm{MgCl}_{2} \cdot 6 \mathrm{H}_{2} \mathrm{O}, 1 \cdot 0 \%$ yeast extract and $0.75 \%$ glycine ( $\mathrm{pH} 7 \cdot 0$ ) for $48 \mathrm{~h}$. The mycelia were then harvested and incubated in $5 \mathrm{ml} \mathrm{P}$ (protoplast) buffer ( $\mathrm{g}$ mycelium $)^{-1}$ containing $1 \mathrm{mg}$ lysozyme $\mathrm{ml}^{-1}$ for $30-60 \mathrm{~min}$ at $30{ }^{\circ} \mathrm{C}$.

For transformation of $S$. roseochromogenes, pFP02 was mixed with $200 \mu \mathrm{l} \mathrm{P}$-buffer containing $1 \times 10^{9} \mathrm{~S}$. roseochromogenes protoplasts and $500 \mu \mathrm{l} \mathrm{T}$ (transformation) buffer containing $50 \%$ (w/v) polyethylene glycol 1000 (Roth). The resulting suspension was plated onto R2YE agar. After incubation for $20 \mathrm{~h}$ at $30^{\circ} \mathrm{C}$, the plates were overlaid with $3 \mathrm{ml}$ of soft R2YE agar containing a total of $500 \mu \mathrm{g}$ thiostrepton, for selection of the recombinant mutants.

After the transformation of $S$. roseochromogenes protoplasts with $\mathrm{pFP} 02$, thiostrepton-resistant colonies were obtained. 
Table 1. Identified ORFs in the biosynthetic gene cluster of clorobiocin

\begin{tabular}{|c|c|c|c|c|}
\hline ORF & $\begin{array}{l}\text { Size of the } \\
\text { product (aa) }\end{array}$ & Similar entity/entities* & $\begin{array}{l}\text { Identity with similar } \\
\text { entity/entities }(\%)\end{array}$ & Accession no. \\
\hline ORF1 & 306 & Fkbl (lysine cyclodeaminase) from Streptomyces hygroscopicus & 37 & AAF86391 \\
\hline ORF2 & 197 & Sarcosine oxidase $\gamma$-subunit from Corynebacterium sp. & 44 & Q46338 \\
\hline ORF3 & 962 & Sarcosine oxidase $\alpha$-subunit from Corynebacterium $\mathrm{sp}$. & 59 & Q46337 \\
\hline ORF4 & 93 & Sarcosine oxidase $\delta$-subunit from Corynebacterium sp. & 66 & Q46336 \\
\hline ORF5 & 406 & Sarcosine oxidase $\beta$-subunit from Corynebacterium $\mathrm{sp}$. & 80 & P40875 \\
\hline ORF6 & 406 & Serine hydroxymethyltransferase from S. coelicolor & 75 & O86565 \\
\hline ORF7 & 218 & Putative transcriptional regulator from $S$. coelicolor & 45 & AL596248.1 \\
\hline ORF8 & 149 & Unknown protein & & \\
\hline ORF9 & 78 & Transposase from S. coelicolor & 68 & AL109949.1 \\
\hline \multirow[t]{2}{*}{ cloE } & 217 & $\operatorname{nov} \boldsymbol{E}(217$ aa) & 82 & \\
\hline & & lmbU protein from $S$. lincolnensis & 43 & S44974 \\
\hline \multirow[t]{2}{*}{ cloF } & 362 & novF $(362$ aa $)$ & 73 & \\
\hline & & Prephenate dehydrogenase from Streptomyces lavendulae & 30 & AAK81837 \\
\hline \multirow[t]{2}{*}{ cloG } & 319 & novG $(318$ aа) $/$ couG $(319$ aа $)$ & $79 / 80$ & \\
\hline & & Regulatory protein (StrR) from Streptomyces glaucescens & 40 & S44506 \\
\hline \multirow[t]{2}{*}{$\operatorname{clo} Y$} & 71 & $\operatorname{cou} Y(71 \mathrm{aa})$ & 81 & \\
\hline & & Hypothetical protein from S. coelicolor & 68 & T36310 \\
\hline \multirow[t]{2}{*}{ cloH } & 600 & novH $(600$ aа) $/ \operatorname{couH}(599$ aа $)$ & $75 / 80$ & \\
\hline & & Peptide synthetase-like protein from Streptomyces antibioticus & 50 & AAG34184.1 \\
\hline \multirow[t]{2}{*}{ cloI } & 407 & novI (407 aa)/couI (407 aa) & $90 / 95$ & \\
\hline & & Cytochrome P450 enzyme from S. antibioticus & 66 & AAG34185.1 \\
\hline \multirow[t]{2}{*}{ cloJ } & 258 & novJ $(262$ aa $) /$ couJ $(258$ aa $)$ & $72 / 77$ & \\
\hline & & 3-Oxoacyl reductase from Bacillus halodurans & 48 & BAB06210 \\
\hline \multirow[t]{2}{*}{ cloK } & 245 & $\operatorname{nov} \boldsymbol{K}(244$ aа) $/ \operatorname{cou} \boldsymbol{K}(245$ aа) & $77 / 81$ & \\
\hline & & Reductase from S. antibioticus & 35 & AAG34182 \\
\hline \multirow[t]{2}{*}{ cloL } & 527 & novL (527 aa)/couL (529 aa) & $86 / 86$ & \\
\hline & & Amide synthetase from $S$. antibioticus & 37 & AAG34183 \\
\hline \multirow[t]{2}{*}{ cloM } & 390 & novM $(379$ aа) $/$ couM $(402$ aа) & $78 / 78$ & \\
\hline & & Glycosyltransferase from Streptomyces argillaceus & 38 & AAC64927 \\
\hline cloN1 & 95 & $\operatorname{couN1}(95 \mathrm{aa}) /$ hypothetical protein & 86 & \\
\hline \multirow[t]{2}{*}{ cloN2 } & 355 & $\operatorname{couN2}(355 \mathrm{aa})$ & 86 & \\
\hline & & $d p s C$ from Streptomyces peucetius & 32 & 1093565 \\
\hline \multirow[t]{2}{*}{ cloN3 } & 376 & $\operatorname{couN3}(373 \mathrm{aa})$ & 81 & \\
\hline & & Acyl-CoA dehydrogenase $(p l t E)$ from $P$. fluorescens & 45 & AAD24879 \\
\hline \multirow[t]{2}{*}{ cloN4 } & 501 & $\operatorname{couN4}(501 \mathrm{aa})$ & 87 & \\
\hline & & Acyl-CoA synthetase $(p l t F)$ from $P$. fluorescens & 45 & AAD24881 \\
\hline \multirow[t]{2}{*}{ cloN5 } & 89 & $\operatorname{couN5}(89 \mathrm{aa})$ & 91 & \\
\hline & & Hypothetical protein ( $p l t L)$ from $P$. fluorescens & 34 & T17418 \\
\hline \multirow[t]{2}{*}{ cloN6 } & 561 & $\operatorname{couN6}(560 \mathrm{aa})$ & 94 & \\
\hline & & Hypothetical protein & & \\
\hline \multirow[t]{2}{*}{ cloN7 } & 278 & $\operatorname{couN7}(281 \mathrm{aa})$ & 82 & \\
\hline & & Putative hydrolase from S. coelicolor & 34 & CAB95984 \\
\hline clo-hal & 524 & Non-haem halogenase from S. lavendulae & 35 & AAK81830 \\
\hline \multirow[t]{2}{*}{ cloP } & 277 & $\operatorname{novP}(262$ aa $) / \operatorname{couP}(276$ aa $)$ & $91 / 89$ & \\
\hline & & O-Methyltransferase III from Streptomyces olivaceus & 58 & CAC32469 \\
\hline $\operatorname{clo} Q$ & 324 & novQ $(323$ aa)/hypothetical protein & 84 & \\
\hline \multirow[t]{2}{*}{ cloR } & 277 & $\operatorname{nov} \boldsymbol{R}(270 \mathrm{aa})$ & 95 & \\
\hline & & Putative aldolase from S. coelicolor & 47 & CAB82026 \\
\hline \multirow[t]{2}{*}{$c l o S$} & 288 & $\operatorname{nov} S(288 \mathrm{aa}) / \operatorname{cou} S(288 \mathrm{aa})$ & $84 / 87$ & \\
\hline & & 4-Ketoreductase from S. antibioticus & 50 & AAD55455 \\
\hline \multirow[t]{2}{*}{ clot $T$} & 336 & $\boldsymbol{n o v} \boldsymbol{T}(336 \mathrm{aa}) / \operatorname{cou} \boldsymbol{T}(336 \mathrm{aa})$ & $82 / 87$ & \\
\hline & & dNDP-glucose-4,6-dehydratase from Streptomyces globisporus & 66 & AAF13998 \\
\hline
\end{tabular}


Table 1 (continued).

\begin{tabular}{|c|c|c|c|c|}
\hline ORF & $\begin{array}{l}\text { Size of the } \\
\text { product }(\mathrm{aa})\end{array}$ & Similar entity/entities* & $\begin{array}{l}\text { Identity with similar } \\
\text { entity/entities (\%) }\end{array}$ & Accession no. \\
\hline \multirow[t]{2}{*}{ cloU } & 420 & $\operatorname{nov} \boldsymbol{U}(420 \mathrm{aa}) / \operatorname{cou} \boldsymbol{U}(420 \mathrm{aa})$ & $88 / 90$ & \\
\hline & & D-Mycarose 3-C-methyltransferase from S. argillaceus & 48 & CAB96549 \\
\hline \multirow[t]{2}{*}{ cloV } & 296 & novV $(297 \mathrm{aa}) / \operatorname{cou} V(296 \mathrm{aa})$ & $89 / 92$ & \\
\hline & & dTDP-glucose synthase from Streptomyces spectabilis & 74 & AAD31796 \\
\hline \multirow[t]{2}{*}{ cloW } & 198 & nov $W(207$ aa $) / \operatorname{cou} W(198 \mathrm{aa})$ & $86 / 91$ & \\
\hline & & dTDP-4-keto-6-deoxyglucose 3,5-epimerase from $S$. glaucescens & 54 & S44236 \\
\hline cloZ & 253 & Hypothetical protein & & \\
\hline$g y r B^{\mathrm{R}}$ & $\begin{array}{l}\text { Partial } \\
\text { sequence }\end{array}$ & $\operatorname{gyr} \boldsymbol{B}^{\mathrm{R}}-\operatorname{nov}$ (novobiocin cluster) $/ \boldsymbol{g} \boldsymbol{y} \boldsymbol{r} \boldsymbol{B}^{\mathrm{R}}-\boldsymbol{c o u}$ (coumermycin cluster) & & \\
\hline
\end{tabular}

* nov genes are from the novobiocin biosynthetic gene cluster of S. spheroides (accession no. AF170880); cou genes are from the coumermycin $\mathrm{A}_{1}$ biosynthetic gene cluster of $S$. rishiriensis (accession no. AF235050).

The single-cross-over mutant RSCO2 was grown in the absence of thiostrepton, allowed to sporulate and then examined for loss of resistance to thiostrepton due to doublecross-over events. Two mutants, named RDCO30 and RDCO32, were examined further. Chromosomal DNA from wild-type $S$. roseochromogenes, as well as from mutants RSCO2, RDCO30 and RDCO32, was digested with SacII and hybridized with a probe containing part of cloR. A band of approximately $1 \cdot 1 \mathrm{~kb}$ in size was detected upon hybridization of the wild-type $S$. roseochromogenes DNA with the probe, whereas hybridization of the chromosomal DNA from mutant strain RDCO30 with the probe produced the expected $2 \cdot 2 \mathrm{~kb}$ band, which corresponded to the in-frame deletion of cloR (Fig. 2).

Complementation of the cloR-defective mutant with Ring A. 3-Dimethylallyl-4-hydroxybenzoic acid (= Ring A of clorobiocin and novobiocin) was obtained by hydrolysis of novobiocin, as described by Kominek \& Sebek (1974). The cloRdefective mutant strain RDCO30 was grown in $50 \mathrm{ml}$ preculture medium (see above) supplemented with $1 \mathrm{mg}$ Ring A. After $48 \mathrm{~h}$ incubation in the pre-culture medium, $5 \mathrm{ml}$ of the culture were inoculated into $50 \mathrm{ml}$ production medium (see above) supplemented with $1 \mathrm{mg}$ Ring A. After 7 days cultivation of the strain in the production medium, secondary metabolites were analysed.

Analysis of secondary metabolites. The bacterial culture $(20 \mathrm{ml})$ was acidified to $\mathrm{pH} 4$ with $\mathrm{HCl}$ and extracted twice with an equal volume of ethyl acetate. After centrifugation, the solvent was evaporated and the dried extract was resuspended in $1 \mathrm{ml}$ methanol. Metabolites were analysed by HPLC using a Multosphere RP18-5 column $(250 \times 4 \mathrm{~mm}$, $5 \mu \mathrm{m}$ ) with a linear gradient from 60 to $100 \%$ methanol in $1 \%$ aqueous formic acid and detection at $340 \mathrm{~nm}$. Authentic clorobiocin (Aventis) was used as the standard.

For preparative isolation of the secondary metabolites, the fractions from HPLC analysis were collected and the solvent was evaporated. The product was analysed by MS and ${ }^{1} \mathrm{H}-$ NMR. Negative fast-atom bombardment (FAB) mass spectra were recorded on a TSQ70 spectrometer (Finnigan) using methanol as the solvent; peaks $\mathrm{A}$ and $\mathrm{B}$ gave identical isotopic peaks, characteristic for substances with one chlorine atom, at $m / z 697$ and $695[\mathrm{M}-\mathrm{H}]^{-}$that corresponded to the clorobiocin standard. The ${ }^{1} \mathrm{H}-\mathrm{NMR}$ spectrum was measured on an AMX 400 spectrometer (Bruker), and peak A gave signals corresponding to those of the clorobiocin standard: $\delta_{\text {p.p.m. }}$
$\left(\mathrm{CD}_{3} \mathrm{OD}, 400 \mathrm{MHz}\right), 7 \cdot 90(\mathrm{~d}, J=9 \cdot 2 \mathrm{~Hz}, \mathrm{H}-5), 7 \cdot 76$ (d, $\left.J=2.5 \mathrm{~Hz}, \mathrm{H}-2^{\prime}\right), 7 \cdot 72\left(\mathrm{dd}, J=8.4 \mathrm{~Hz}, 2.5 \mathrm{~Hz}, \mathrm{H}-6^{\prime}\right), 7 \cdot 33$ (d, $J=9.2 \mathrm{~Hz}, \mathrm{H}-6), 6.90\left(\mathrm{~d}, J=3.6 \mathrm{~Hz}, \mathrm{H}-3^{\prime \prime \prime}\right), 6.84$ (d, $J=8.4 \mathrm{~Hz}$, H-5'), 5.94 (d, J=3.6 Hz, H-4"'), 5.73 (d, J=1.8 Hz, H-1"), 5.71 $\left(\mathrm{dd}, J=10 \cdot 3 \mathrm{~Hz}, J=2 \cdot 9 \mathrm{~Hz}, \mathrm{H}-3^{\prime \prime}\right), 5 \cdot 35$ (broad t, $J=7 \cdot 1 \mathrm{~Hz}$, H-8'), 4.34 (t, $\left.J=2.7 \mathrm{~Hz}, \mathrm{H}-2^{\prime \prime}\right), 3.72$ (d, $\left.J=10 \cdot 3 \mathrm{~Hz}, \mathrm{H}-4^{\prime \prime}\right)$,

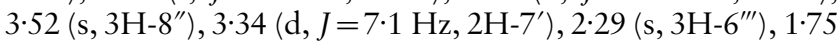
(s, 3H-11'), $1 \cdot 74$ (s, 3H-10'), 1.35 (s, 3H-7"), 1.18 (s, 3H-6").

\section{RESULTS}

\section{Cloning and sequencing of the clorobiocin biosynthetic gene cluster}

We have previously cloned the novobiocin (Steffensky et al., 2000a) and coumermycin $\mathrm{A}_{1}$ (Wang et al., 2000) biosynthetic gene clusters (Fig. 1). In the novobiocin cluster, novT encodes a dTDP-glucose 4,6-dehydratase involved in the biosynthesis of the deoxysugar moiety of novobiocin; novL encodes the novobiocic acid synthetase that catalyses the formation of the amide bond between Ring A and Ring B of novobiocin (Fig. 1) (Steffensky et al., 2000b). Similar reactions to these were expected to be involved in clorobiocin biosynthesis. Therefore, Southern hybridizations of genomic DNA from the clorobiocin producer $S$. roseochromogenes were carried out with probes for novT and novL; each hybridization resulted in a single band.

A cosmid library from $S$. roseochromogenes was constructed in SuperCos-1 and screened with the novT and novL probes. The hybridizing cosmids were analysed by restriction mapping. Four different but overlapping cosmids were obtained which covered a continuous $55 \mathrm{~kb}$ region of the chromosome. Cosmid K1F2 was sequenced on both strands. From the sequence of this cosmid, 36 ORFs were identified; 27 of these showed striking similarity to genes of the novobiocin and/or coumermycin $A_{1}$ biosynthetic gene clusters. In addition, a partial sequence of the aminocoumarin-resistance gene $\left(g y r B^{\mathrm{R}}\right)$ was found at the 3' end of the clorobiocin gene cluster. Strikingly, in the gene clusters of coumermycin $\mathrm{A}_{1}$, novobiocin and clorobiocin, the corresponding 


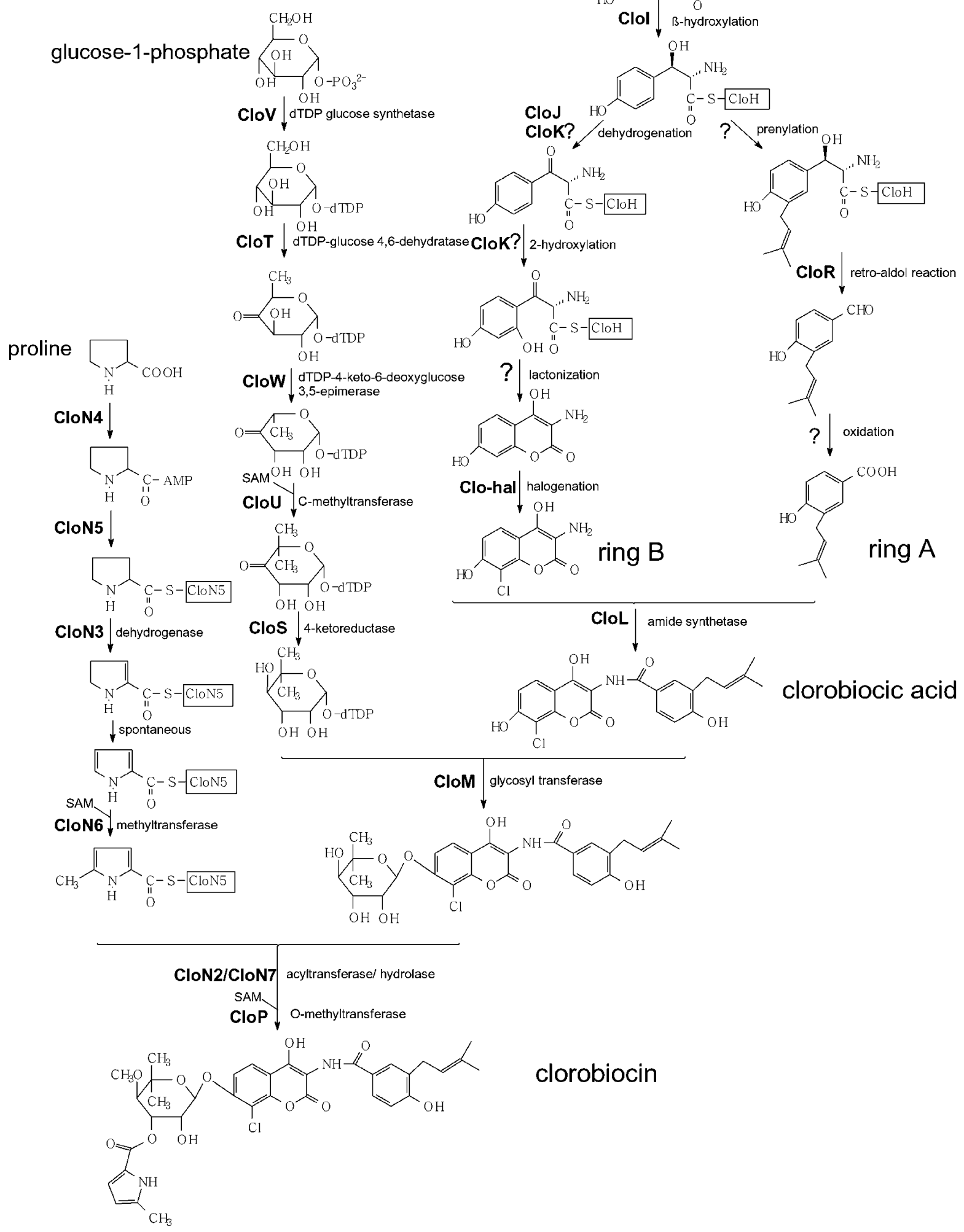

Fig. 3. Hypothetical biosynthetic pathway for clorobiocin. 
ORFs were arranged in exactly the same order and oriented in the same direction (Fig. 1).

Table 1 lists the homologies found between the genes in cosmid K1F2 and the genes of the novobiocin and coumermycin $\mathrm{A}_{1}$ clusters, as well as the homologies of the K1F2 genes to other sequences found in GenBank. The sequence of cosmid K1F2 has been deposited in GenBank under accession no. AF329398.

\section{Genes involved in the biosynthesis of the deoxysugar moiety (Ring C)}

At the $3^{\prime}$ end of the clorobiocin gene cluster, five ORFs with high homology to genes involved in the deoxysugar biosynthesis were discovered (cloSTUVW) (Table 1). Homologous genes are found in the same position in the gene clusters of novobiocin (novSTUVW) and coumermycin $\mathrm{A}_{1}$ (couSTUVW) (Fig. 1). Based upon their homology to known genes involved in deoxysugar biosynthesis, we previously assigned these genes to the five steps required for the biosynthesis of the deoxysugar moiety (Fig. 3); functional proof for this hypothesis was provided by an inactivation experiment with nov $T$ in the novobiocin producer S. spheroides (Steffensky et al., 2000a). The presence of these genes in the clorobiocin gene cluster provides additional support to our previous functional assignment of these genes.

O-Methylation at position 4 of the deoxysugar moiety is regarded as the last step in aminocoumarin biosynthesis (Fig. 3) (Queener et al., 1978). The genes cloP, novP and couP, situated in the same relative position of their respective gene clusters, show homology to genes encoding $\mathrm{O}$-methyltransferases and may be assigned to this reaction. CouP has recently been proven to be responsible for the methylation of 4-OH of the deoxysugar (Li et al., 2002).

\section{Genes presumably involved in the formation of the pyrrole ring}

Clorobiocin and coumermycin $\mathrm{A}_{1}$ contain pyrrole carboxylic acid rings attached to position 3 of their deoxysugar moieties (Fig. 1). Novobiocin contains a carbamyl group at the corresponding position.

These structural similarities and differences between the three antibiotics are reflected in the organization of their respective gene clusters (Fig. 1). Downstream of the glycosyltransferase gene nov $M$, the novobiocin cluster contains a gene $(n o v N)$ with homology to those encoding carbamoyl transferases, whereas in the same relative position of the clorobiocin and coumermycin clusters, a group of seven genes is found (cloN1-N7 or couN1-N7, respectively) which show very high homology between the two clusters (on average $87 \%$ identity). These genes can be assigned to pyrrole biosynthesis, which has been elucidated on the genetic and biochemical level: cloN3, cloN4 and cloN5 show sequence similarities to $p l t E, p l t F$ and $p l t L$, respectively, which are involved in the biosynthesis of the pyrrole moiety of pyoluteorin in Pseudomonas fluorescens Pf-5 (Nowak-Thompson et al., 1999) and to redW, redM and red $\mathrm{O}$, respectively, which are involved in the biosynthesis of the pyrrole moiety of undecylprodiginin in Streptomyces coelicolor (Cerdeno et al., 2001). PltF and RedM convert L-proline into its acyl adenylate (Fig. 3) and the small proteins PltL and RedO act as peptidyl carrier proteins (PCPs) (Thomas et al., 2002). Therefore, the same functions may be assigned to the homologous proteins CloN4 and CloN5, respectively. CloN3, like CouN3, PltE and RedW, shows homology to flavinedependent acyl-coenzyme A dehydrogenases. PltE and RedW catalyse the dehydrogenation of the PCP-bound proline (Thomas et al., 2002). The resulting pyrroline derivative (presumably $\Delta^{2}$ ) undergoes spontaneous oxidation to the aromatic pyrrole derivative (Fig. 3).

CloN6 (CouN6) belongs to the BchE-like/methyltransferase subgroup of radical SAM proteins, which has recently been identified using bioinformatic techniques (Sofia et al., 2001). We suggest that CloN6 catalyses the transfer of a methyl group to position 5 of the pyrrole2-carboxylic acid. cloN2 (couN2) shares homology with $d p s C$, which encodes an enzyme with acyltransferase activity. cloN7 and/or cloN2 may be involved in the transfer of the activated pyrrole-2-carboxylic acid to the $3-\mathrm{OH}$ of the deoxysugar moiety. The small ORF cloN1 (encoding $95 \mathrm{aa}$ ) does not show homology to other database entries, and its function remains unknown at present.

\section{Genes presumably involved in the biosynthesis of the aminocoumarin ring (Ring B)}

The genes for the biosynthesis of the characteristic aminocoumarin ring must be present in all three gene clusters; hence, a comparison of the three gene clusters presents an obvious method for identifying possible candidate genes involved in the biosynthesis of this ring. In the clorobiocin cluster, cloHIJK showed, on average, $85 \%$ homology to the corresponding genes in the novobiocin (novHIJK) and coumermycin $\mathrm{A}_{1}$ (couHIJK) clusters (Table 1). It appears likely that the products of these genes are involved in the formation of the aminocoumarin ring from tyrosine (see Discussion).

Clorobiocin contains a chlorine atom at position 8 of the aminocoumarin ring, whereas novobiocin and coumermycin $A_{1}$ contain a methyl group at the same position (Fig. 1). This structural difference of the antibiotics is perfectly reflected in the organization of their gene clusters: the novobiocin and coumermycin clusters contain a C-methyltransferase gene, nov $\mathrm{O}$ or $\operatorname{couO}$, respectively, whereas in the clorobiocin cluster, clo-hal, a homologue of non-haem halogenase genes, is found at the same relative position.

\section{Genes presumably involved in the biosynthesis of the 3-dimethylallyl-4-hydroxybenzoic acid (Ring A)}

Clorobiocin and novobiocin contain a prenylated 4hydroxybenzoate moiety (Ring A). Coumermycin $A_{1}$ 

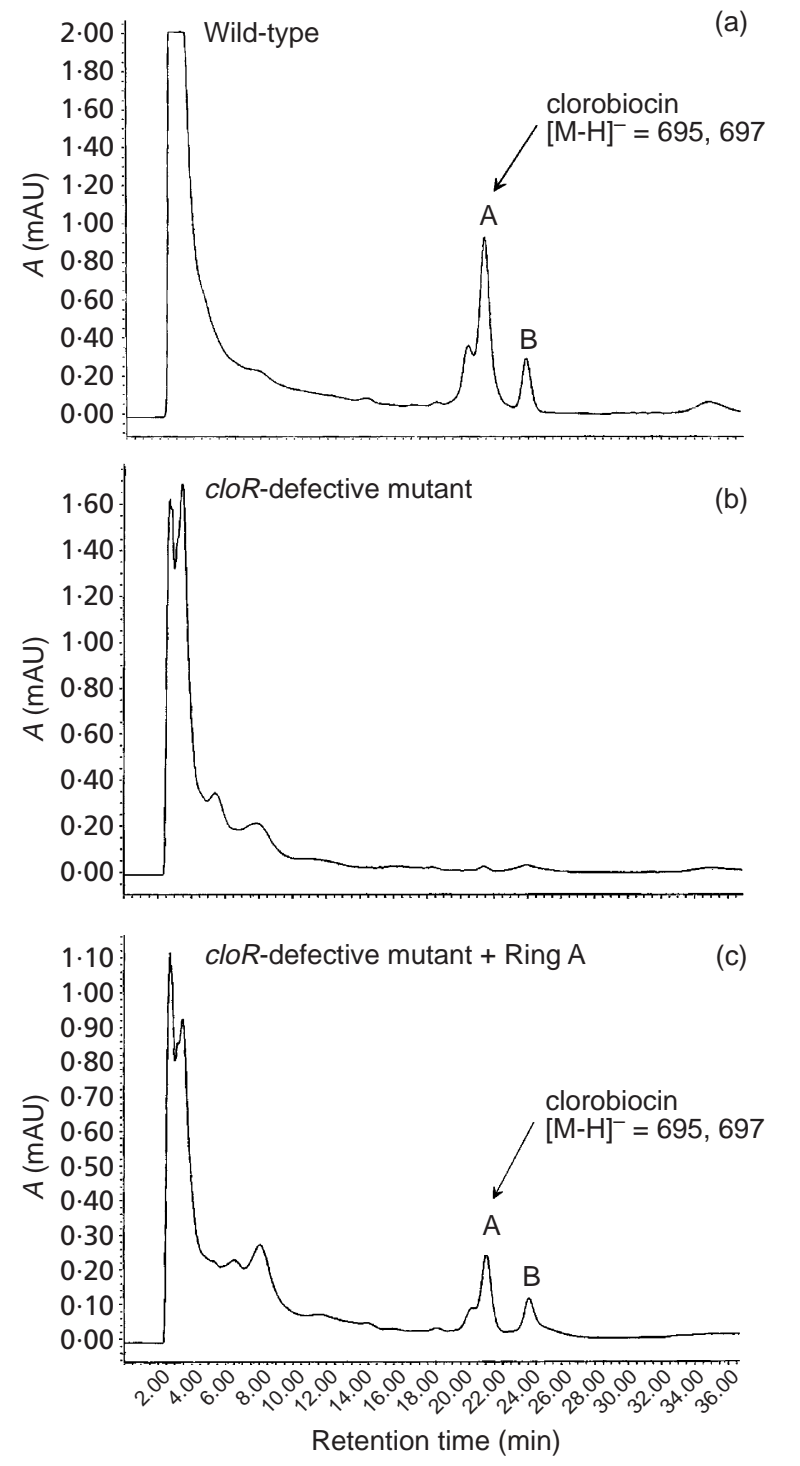

Fig. 4. HPLC analysis of the secondary metabolites produced by (a) wild-type S. roseochromogenes DS 12.976, (b) the cloRdefective mutant and (c) the cloR-defective mutant complemented with Ring A (3-dimethylallyl-4-hydroxybenzoic acid). Absorbance values are in milli arbitrary units (mAU).

contains a pyrrole dicarboxylic acid moiety instead, linking the two aminocoumarin rings of this molecule (Fig. 1). The aromatic nucleus of Ring A of clorobiocin and novobiocin is derived from tyrosine (Bunton et al., 1963; Kominek \& Sebek, 1974), but the exact reaction sequence is unknown.

Sequencing of the clorobiocin gene cluster revealed two genes that are also present in the novobiocin cluster, but which are not present in the coumermycin cluster, i.e. $c l o Q$ and $c l o R$. This fact drew us to the hypothesis that these genes may be involved in Ring A biosynthesis.

cloQ and cloR, like nov $Q$ and nov $R$, show transcriptional coupling (i.e. the stop codon of $c l o Q$ is fused with the start codon of $c l o R$ ) and are likely to be transcribed as a single operon. Unusually large intergenic regions are found upstream and downstream of cloQR (1001 and $830 \mathrm{bp}$, respectively). CloR has $47 \%$ identity to a putative aldolase from $S$. coelicolor. cloQ did not show homologies to other genes in the database, with the exception of novQ.

\section{Generation of a cloR-defective mutant}

To test whether cloR was indeed involved in Ring A biosynthesis, a gene inactivation experiment was carried out. An inactivation vector carrying a thiostreptonresistance gene ( $\mathrm{pFP} 02$ ) was constructed, in which the structural gene cloR was disrupted by an in-frame deletion (Fig. 2). The deletion mutant, RDCO30, was subsequently cultured and the ethyl acetate extract of the culture was examined by HPLC for secondary metabolites. As shown in Fig. 4, the production of clorobiocin was abolished in this mutant. Another thiostrepton-sensitive strain obtained in the screening for double-cross-over mutants, RDCO32, represented a reversion to the wild-type (Fig. 2), and showed clorobiocin production identical to that of the wild-type strain (data not shown).

\section{Feeding of Ring A to the cloR-defective mutant}

To restore clorobiocin biosynthesis in the cloR-defective mutant strain RDCO30, Ring A (3-dimethylallyl-4hydroxybenzoic acid) was added to the culture medium of this strain. This led to the reconstitution of clorobiocin production (Fig. 4), to one-third the level of the wild-type strain. The identity of this product was confirmed by MS and ${ }^{1} \mathrm{H}-\mathrm{NMR}$, in a comparison with authentic clorobiocin.

Besides the major peak for clorobiocin (peak A in Fig. 4), a minor peak of identical mass (peak B) was detected both in the wild-type and the complemented mutant strain. This substance is likely to represent an isomer of clorobiocin, possibly carrying the pyrrole carboxylic acid moiety in position 2 instead of position 3 of the deoxysugar. Such isomers have been reported previously for novobiocin (Hinman et al., 1957).

\section{Genes involved in the linkage of Rings A, B and C of clorobiocin}

Attachment of the deoxysugar to the 7-OH group of the aminocoumarin ring requires very similar glycosyl transferases in clorobiocin, novobiocin and coumermycin $\mathrm{A}_{1}$ biosynthesis; indeed, three very similar putative glycosyltransferase genes, cloM, nov $M$ and couM, are found at the same relative position in all three gene clusters.

In clorobiocin and novobiocin, the aminocoumarin moiety (Ring B) and the prenylated 4-hydroxybenzoate moiety (Ring A) are linked by an amide bond (Fig. 1). It has been demonstrated that the enzyme NovL catalyses 
this reaction, i.e. the adenylation of the substituted benzoyl moiety and its transfer to the amino group (Steffensky et al., 2000b). cloL shows high homology to novL and is most probably involved in the formation of the amide bond of clorobiocin.

\section{Resistance and regulatory genes}

Downstream of the deoxysugar biosynthesis genes cloSTUVW in the clorobiocin cluster, and similarly at the corresponding position of the novobiocin and coumermycin clusters, a gene encoding an aminocoumarin-resistant gyrase B subunit $\left(g y r B^{\mathrm{R}}\right)$ is located. This gene has previously been identified as the principal novobiocin-resistance gene in the novobiocin producer S. spheroides (Thiara \& Cundliffe, 1988).

cloG, nov $G$ and $\operatorname{cou} G$ are homologous to strR, a regulatory gene from the streptomycin cluster. Streptomycin biosynthesis is known to be regulated by $\gamma$ butyrolactones (Horinouchi \& Beppu, 1995). It may, therefore, be speculated that $\gamma$-butyrolactones are involved in the regulation of the biosynthesis of clorobiocin and other aminocoumarin antibiotics.

cloE has homology to the $\operatorname{lmbU}$ gene of the lincomycin biosynthetic gene cluster of Streptomyces lincolnensis 78-11. It was suggested that LmbU may have a regulatory function, but no experimental evidence is available so far to support this (Peschke et al., 1995).

\section{Genes with unknown function}

At present, no function can be suggested for the small ORFs cloY and cloN1, which have homologues in the coumermycin $\mathrm{A}_{1}$ cluster, and for cloZ, which has no homologues in other clusters.

\section{DISCUSSION}

In this study, we have cloned and sequenced the clorobiocin biosynthetic gene cluster which spans approximately $31 \mathrm{~kb}$ and comprises 27 ORFs. The suggested function of these ORFs in clorobiocin biosynthesis is depicted in Fig. 3.

The primary metabolic genes found at the $5^{\prime}$ end of the clorobiocin cluster (Table 1 ) suggest that $c l o E$ represents the $5^{\prime}$ border of the cluster. The gene adjacent to cloE, i.e. ORF9, encodes a putative transposase, and it may be speculated that this gene is related to the introduction of the cluster into the $S$. roseochromogenes genome. Also at the $3^{\prime}$ end, downstream of $g y r B^{\mathrm{R}}$, primary metabolic genes were found, suggesting that the sequence depicted in Fig. 1 comprises all biosynthetic genes of the clorobiocin cluster. However, it cannot be excluded that additional biosynthetic enzymes for clorobiocin formation are encoded at different loci of the genome.

The clorobiocin cluster contains the genes cloHIJK, for which homologues exist in the novobiocin cluster (novHIJK) and the coumermycin cluster (couHIJK). NovH has recently been shown to activate tyrosine by covalent binding to the 4-phosphopantetheinyl cofactor, and the P450 enzyme NovI catalyses the $\beta$-hydroxylation of the activated tyrosine (Chen \& Walsh, 2001). A central, unresolved question in aminocoumarin biosynthesis is how activated $\beta$-hydroxy tyrosine is then converted to the coumarin ring, especially how the ring oxygen is introduced.

cloJ and cloK, the genes immediately downstream of $c l o H$ and cloI, are homologous to novJK and couJK of the novobiocin and coumermycin clusters, respectively. The detection of $c l o J$ and $c l o K$ in the clorobiocin cluster, and the homologous simJ1 and $\operatorname{sim} K$ genes in the biosynthetic gene cluster of the aminocoumarin antibiotic simocyclinone (Galm et al., 2002; Trefzer et al., 2002), now strongly supports the hypothesis that these genes are indeed related to aminocoumarin biosynthesis. cloJ shows homology to 3-oxo-acyl-[ACP] reductases and may likely be involved in the oxidation of a $\beta$ hydroxy-tyrosyl to a $\beta$-keto-tyrosyl intermediate (Fig. 3). Also, cloK shows homology to oxidoreductases, but this homology is not very high (mean of $35 \%$ on the amino acid level). Chen \& Walsh (2001) speculated that NovJ and NovK may act together to oxidize the $\beta$ hydroxyl function to a keto group. The unresolved step in the postulated Ring B biosynthesis, however, is the hydroxylation of the activated tyrosyl derivative in position 2 of the aromatic nucleus (Fig. 3). Bunton et al. (1963) had reported that the ring oxygen of the aminocoumarin may be derived from the carboxyl group of tyrosine rather than from molecular oxygen. This was recently disproven by Holzenkämpfer \& Zeeck (2002), who showed that the ring oxygen of the aminocoumarin moiety of simocyclinone is in fact derived from molecular oxygen. Therefore, coumarin ring formation most likely proceeds via the 2-hydroxylation of a tyrosine derivative. Chen \& Walsh (2001) had speculated that the predicted flavine dioxygenase NovC, encoded by a gene near the novobiocin cluster, may catalyse this reaction. An important finding of our study is that no nov C homologue was detected in or near the clorobiocin gene cluster. Likewise, no nov C homologue was detected in the simocyclinone cluster (Galm et al., 2002; Trefzer et al., 2002). We therefore suggest that nov $C$ is not related to aminocoumarin biosynthesis. The enzyme responsible for the 2-hydroxylation of the $\beta$ keto-tyrosyl intermediate remains unknown at present. Whether cloK is involved in this or another reaction of aminocoumarin biosynthesis has yet to be demonstrated.

Halogenation of the aminocoumarin ring may occur after ring formation, as depicted in Fig. 3, or at an earlier stage.

The prenylated 4-hydroxybenzoate moiety (Ring A) of clorobiocin and novobiocin is formed from tyrosine (Kominek \& Sebek, 1974) and an isoprenoid precursor, but this reaction sequence is also unknown. The biosynthesis of this moiety requires: (i) the assembly of the isoprenoid precursor (probably dimethylallyl diphosphate) via the methylerythritol phosphate pathway 
(Li et al., 1998); (ii) the conversion of the phenylpropanoid compound tyrosine to a benzoic acid derivative; and (iii) the prenylation of the aromatic nucleus in a prenyltransferase reaction. The conversion of the phenylpropanoid intermediate to a benzoic acid derivative may proceed by a mechanism analogous to the oxidation of fatty acids, as demonstrated in Streptomyces maritimus (Hertweck \& Moore, 2000). Alternatively, this conversion may occur by retro-aldol cleavage of a 3-hydroxylated phenylpropanoid compound, as found in P. fluorescens (Gasson et al., 1998) and Amycolatopsis sp. (Achterholt et al., 2000). Retro-aldol cleavage would result in a benzaldehyde derivate, which would subsequently be oxidized to the benzoic acid derivative.

In the clorobiocin cluster, we could not detect genes similar to those for the $\beta$-oxidation of fatty acids. We did find, however, the gene $c l o R$ which showed homology to a putative aldolase from $S$. coelicolor. Comparison of the gene clusters of clorobiocin, novobiocin and coumermycin $\mathrm{A}_{1}$ led to the hypothesis that this gene may be involved in Ring A biosynthesis. Inactivation of the enzyme led to an abolishment of clorobiocin production. When the mutant was complemented with the prenylated 4-hydroxybenzoate moiety (=Ring A), clorobiocin production was restored. This proved that $c l o R$ is indeed involved in Ring A biosynthesis. We suggest that the formation of Ring A of clorobiocin may proceed via a retro-aldol reaction catalysed by CloR, i.e. by a mechanism different from the elucidated benzoic acid biosynthesis in S. maritimus (Hertweck \& Moore, 2000).

The substrate of CloR may be an enzyme-bound prenylated $\beta$-hydroxytyrosine, as suggested by Chen $\&$ Walsh (2001) and depicted in Fig. 3. Alternatively, prenylated 4-hydroxyphenylpyruvate may be the substrate of CloR, since this compound was detected as the product of a prenyltransferase of the novobiocin producer S. spheroides (Steffensky et al., 1998). Further studies are now in progress to investigate these steps in clorobiocin biosynthesis.

The cloning and sequencing of the clorobiocin gene cluster has completed the genetic information on the biosynthesis of three 'classical' aminocoumarin antibiotics, namely novobiocin, clorobiocin and coumermycin $A_{1}$. Comparison of the three gene clusters revealed a strikingly stringent correspondence between the structures of the antibiotics and the organization of the biosynthetic genes, unprecedented so far in any class of natural products outside the polyketide and the peptide antibiotics. For each structural moiety of the aminocoumarin antibiotics, the biosynthetic genes are grouped together, resulting in a 'modular' structure of the clusters. The orders of the modules and the order of the genes within each module are perfectly identical for the three 'classical' aminocoumarins, and nearly all of the genes within the clusters are orientated in the same direction. The comparison of the three clusters greatly facilitates the prediction of functions for the different genes. As an example, cloR was recognized by such a comparison as a candidate gene for the biosynthesis of the prenylated 4-hydroxybenzoate moiety of clorobiocin, and this was experimentally proven by an inactivation and complementation experiment. The similarity between the three clusters also provides excellent opportunities for the production of hybrid aminocoumarins by genetic methods.

\section{ACKNOWLEDGEMENTS}

We thank Aventis for the generous gifts of S. roseochromogenes DS 12.976 and authentic clorobiocin. This work was supported by a grant from the Deustche Forschunggemeinschaft (to L. Heide and S.-M. Li) and by the Fonds der Chemischen Industrie.

\section{REFERENCES}

Achterholt, S., Priefert, H. \& Steinbuchel, A. (2000). Identification of Amycolatopsis sp. strain HR167 genes, involved in the bioconversion of ferulic acid to vanillin. Appl Microbiol Biotechnol 54, 799-807.

Ali, J. A., Jackson, A. P., Howells, A. J. \& Maxwell, A. (1993). The 43-kilodalton N-terminal fragment of the DNA gyrase B protein hydrolyzes ATP and binds coumarin drugs. Biochemistry 32, 2717-2724.

Berger, J. \& Batcho, A. D. (1978). Coumarin-glycoside antibiotics. J Chromatogr Libr 15, 101-159.

Bunton, C. A., Kenner, G. W., Robinson, M. J. T. \& Webster, B. R. (1963). Experiments related to the biosynthesis of novobiocin and other coumarins. Tetrahedron 19, 1001-1010.

Cerdeno, A. M., Bibb, M. J. \& Challis, G. L. (2001). Analysis of the prodiginine biosynthesis gene cluster of Streptomyces coelicolor A3(2): new mechanisms for chain initiation and termination in modular multienzymes. Chem Biol 8, 817-829.

Chen, H. \& Walsh, C. T. (2001). Coumarin formation in novobiocin biosynthesis: $\beta$-hydroxylation of the aminoacyl enzyme tyrosyl-SNovH by a cytochrome P450 NovI. Chem Biol 8, 301-312.

Galm, U., Schimana, J., Fiedler, H. P., Schmidt, J., Li, S.-M. \& Heide, L. (2002). Cloning and analysis of the simocyclinone biosynthetic gene cluster of Streptomyces antibioticus Tü 6040. Arch Microbiol 178, 102-114.

Gasson, M. J., Kitamura, Y., McLauchlan, W. R., Narbad, A., Parr, A. J., Parsons, E. L. H., Payne, J., Rhodes, M. J. C. \& Walton, N. J. (1998). Metabolism of ferulic acid to vanillin. A bacterial gene of the enoyl-SCoA hydratase/isomerase superfamily encodes an enzyme for the hydration and cleavage of a hydroxycinnamic acid SCoA thioester. J Biol Chem 273, 4163-4170.

Hertweck, C. \& Moore, B. S. (2000). A plant-like biosynthesis of benzoyl-CoA in the marine bacterium Streptomyces maritimus. Tetrahedron 56, 9115-9120.

Hinman, J. W., Caron, E. L \& Hoeksema, H. (1957). Novobiocin. V. Carbamyl migration and isonovobiocin. J Am Chem Soc 79, 5321-5322.

Holzenkämpfer, M. \& Zeeck, A. (2002). Biosynthesis of simocyclinone D8 in an ${ }^{18} \mathrm{O}_{2}$-rich atmosphere. J Antibiot (Tokyo) 55, 341-342.

Hooper, D. C., Wolfson, J. S., McHugh, G. L., Winters, M. B. \& Swartz, M. N. (1982). Effects of novobiocin, coumermycin A1, clorobiocin, and their analogs on Escherichia coli DNA gyrase and bacterial growth. Antimicrob Agents Chemother 22, 662-671.

Horinouchi, S. \& Beppu, T. (1995). Autoregulators. Biotechnology 28, 103-119. 
Kieser, T., Bibb, M. J., Buttner, M. J., Chater, K. F. \& Hopwood, D. A. (2000). Practical Streptomyces Genetics. Norwich: John Innes Foundation.

Kominek, L. A. \& Sebek, O. K. (1974). Biosynthesis of novobiocin and related coumarin antibiotics. Dev Ind Microbiol 15, 60-69.

Lafitte, D., Lamour, V., Tsvetkov, P. O., Makarov, A. A., Klich, M., Deprez, P., Moras, D., Briand, C. \& Gilli, R. (2002). DNA gyrase interaction with coumarin-based inhibitors: the role of the hydroxybenzoate isopentenyl moiety and the $5^{\prime}$-methyl group of the noviose. Biochemistry 41, 7217-7223.

Lewis, R. J., Singh, O. M., Smith, C. V., Skarzynski, T., Maxwell, A., Wonacott, A. J. \& Wigley, D. B. (1996). The nature of inhibition of DNA gyrase by the coumarins and the cyclothialidines revealed by X-ray crystallography. EMBO J 15, 1412-1420.

Li, S.-M., Hennig, S. \& Heide, L. (1998). Biosynthesis of the dimethylallyl moiety of novobiocin via a non-mevalonate pathway. Tetrahedron Lett 39, 2717-2720.

Li, S.-M., Westrich, L., Schmidt, J., Kuhnt, C. \& Heide, L. (2002). Methyltransferase genes in Streptomyces rishiriensis: new coumermycin derivatives from gene-inactivation experiments. Microbiology 148, 3317-3326.

Lombo, F., Siems, K., Brana, A. F., Mendez, C., Bindseil, K. \& Salas, J. A. (1997). Cloning and insertional inactivation of Streptomyces argillaceus genes involved in the earliest steps of biosynthesis of the sugar moieties of the antitumor polyketide mithramycin. J Bacteriol 179, 3354-3357.

Mancy, D., Ninet, L. \& Preud Homme, J. (1974). Antibiotic 18. 631 RP. United States Patent Office, no. 3793147.

Maxwell, A. (1993). The interaction between coumarin drugs and DNA gyrase. Mol Microbiol 9, 681-686.

Maxwell, A. (1999). DNA gyrase as a drug target. Biochem Soc Trans 27, 48-53.

Nowak-Thompson, B., Chaney, N., Wing, J. S., Gould, S. J. \& Loper, J. E. (1999). Characterization of the pyoluteorin biosynthetic gene cluster of Pseudomonas fluorescens Pf-5. J Bacteriol 181, 2166-2174.

Peschke, U., Schmidt, H., Zhang, H. Z. \& Piepersberg, W. (1995). Molecular characterization of the lincomycin-production gene cluster of Streptomyces lincolnensis 78-11. Mol Microbiol 16, 1137-1156.

Queener, S. W., Sebek, O. K. \& Vezina, C. (1978). Mutants blocked in antibiotic synthesis. Annu Rev Microbiol 32, 593-636.
Sambrook, J. \& Russell, D. (2001). Molecular Cloning: a Laboratory Manual, 3rd edn. Cold Spring Harbor, NY : Cold Spring Harbor Laboratory.

Sofia, H. J., Chen, G., Hetzler, B. G., Reyes-Spindola, J. F. \& Miller, N. E. (2001). Radical SAM, a novel protein superfamily linking unresolved steps in familiar biosynthetic pathways with radical mechanisms: functional characterization using new analysis and information visualization methods. Nucleic Acids Res 29, 10971106.

Steffensky, M., Li, S.-M., Vogler, B. \& Heide, L. (1998). Novobiocin biosynthesis in Streptomyces spheroides: identification of a dimethylallyl diphosphate: 4-hydroxyphenylpyruvate dimethylallyl transferase. FEMS Microbiol Lett 169, 69-74.

Steffensky, M., Mühlenweg, A., Wang, Z. X., Li, S.-M. \& Heide, L. (2000a). Identification of the novobiocin biosynthetic gene cluster of Streptomyces spheroides NCIB 11891. Antimicrob Agents Chemother 44, 1214-1222.

Steffensky, M., Li, S.-M. \& Heide, L. (2000b). Cloning, overexpression, and purification of novobiocic acid synthetase from Streptomyces spheroides NCIMB 11891. J Biol Chem 275, 21754-21760.

Thiara, A. S. \& Cundliffe, E. (1988). Cloning and characterization of a DNA gyrase B gene from Streptomyces sphaeroides that confers resistance to novobiocin. EMBO J 7, 2255-2259.

Thomas, M. G., Burkart, M. D. \& Walsh, C. T. (2002). Conversion of L-proline to pyrrolyl-2-carboxyl-S-PCP during undecylprodigiosin and pyoluteorin biosynthesis. Chem Biol 9, 171-184.

Trefzer, A., Pelzer, S., Schimana, J., Stockert, S., Bihlmaier, C., Fiedler, H. P., Welzel, K., Vente, A. \& Bechthold, A. (2002). Biosynthetic gene cluster of simocyclinone, a natural multihybrid antibiotic. Antimicrob Agents Chemother 46, 1174-1182.

Tsai, F. T., Singh, O. M., Skarzynski, T. \& 9 other authors (1997). The high-resolution crystal structure of a $24-\mathrm{kDa}$ gyrase $\mathrm{B}$ fragment from E. coli complexed with one of the most potent coumarin inhibitors, clorobiocin. Proteins 28, 41-52.

Wang, Z. X., Li, S.-M. \& Heide, L. (2000). Identification of the coumermycin A(1) biosynthetic gene cluster of Streptomyces rishiriensis DSM 40489. Antimicrob Agents Chemother 44, 3040-3048.

Received 28 May 2002; revised 29 July 2002; accepted 22 August 2002. 